eISSN: 2659-6482

DOI: https://doi.org/10.14201/pmrt.19717

\title{
PROGRAMACIÓN DE LA UNIDAD DE MÚSICA DE LA GUARDIA REAL ENTRE 1973 Y 2010
}

\section{Programming of the Music Unit of the Royal Guard between 1973 and 2010}

\section{Eva FOLCH MARTÍNEZ}

Ministerio de Defensa

efolmar@et.mde.es

RESUMEN: En la actualidad subsisten pocas bandas, tanto civiles como militares, pero a cambio tienen un excelente nivel de ejecución, del que la Música de la Guardia Real es paradigma, por eso se ha utilizado la programación de ésta para realizar este estudio científico. Las programaciones de la Música de la Guardia Real, son un excelente ejemplo para otras agrupaciones musicales; razón por la cual se podrán basar conocimientos de programación sobre esta dilatada recopilación.

En su historia, la Música de la Guardia Real, antigua de Alabarderos ha tenido más de quince directores que en el transcurso de los años, han programado infinidad de conciertos. En este estudio sobre la programación de esta agrupación musical, solo hemos abarcado los conciertos programados por tres de los directores que estuvieron al frente en esta unidad entre 1973 y 2010. Estos programas han sido analizados, según su año de publicación, el género musical al que pertenece cada pieza, la naturaleza del evento, si fue un concierto militar o cívico-militar, si tuvo repercusión internacional o solo nacional y en qué ciudad tuvo lugar, además de una pequeña síntesis de cuáles fueron las piezas más interpretadas en cada periodo.

Durante este tiempo, una nueva generación de grandes directores que comenzó con José López Calvo (1973-1988), seguido de Francisco Grau Vegara (1988-2008) y Antonio Sendra Cebolla (2008-2010), supieron llevar a buen término el desarrollo del arte musical, llegando a ser protagonistas principales de la vida musical en Madrid. Manteniendo las tradiciones de 
los Relevos de Palacio, actos protocolarios, desfiles, ciclos de conciertos, giras y demás actividades que tanta labor socio-cultural desarrollaron. Estos directores han sabido elevar al lugar que se merece la música de la Guardia Real, adaptándose a los tiempos que corren y cambiando el repertorio de nuestra banda sinfónica que antiguamente se basaba en transcripciones de orquesta, pasando a interpretar composiciones originales para banda, lo cual da unas sonoridades de planos sonoros mucho más logrados. Estos compositores-directores que han estado al frente de la agrupación, tal y como podemos observar en los programas de mano de los conciertos cívicomilitares que hemos recogido y analizado, han compaginado la dirección con la composición, estrenando diferentes obras con esta agrupación. Esta Música siempre ha sido un referente para todos los directores militares que han deseado llegar algún día a ser director de esta prestigiosa banda y también es una imagen a seguir por bandas civiles, pues en la historia de España siempre ha sido una representación extraordinaria.

Palabras clave: Música militar; repertorio militar; banda militar

ABSTRACT: Currently there are few bands, both civilian and military, but in return they have an excellent level of execution, of which the Music of the Royal Guard is a paradigm, that is why the program has been used to carry out this scientific study. The schedules of the Music of the Royal Guard, are an excellent example for other musical groups; which is why you can base programming knowledge on this extensive compilation.

In its history, the Music of the Royal Guard, formerly of Alabarderos, has had more than fifteen directors who, over the years, have programmed countless concerts. In this study on the programming of this musical group, we have only covered the concerts programmed by three of the directors who were at the front in this unit between 1973 and 2010. These programs have been analyzed, according to their year of publication, the musical genre to which each piece belongs, the nature of the event, whether it was a military or civic-military concert, whether it had international or only national impact and in what city it took place, besides a brief synthesis of which were the most interpreted pieces in each period.

During this time, a new generation of great directors those began with José López Calvo (1973-1988), followed by Francisco Grau Vegara (19882008) and Antonio Sendra Cebolla (2008-2010), and were able to successfully complete the development of the musical art, becoming the main protagonists of musical life in Madrid. Keeping the following traditions; relays of the Palace, ceremonial events, parades, concert cycles, tours and other activities that so much socio-cultural work developed. These directors have managed to raise to the place that deserves the music of the Royal Guard, adapting 
to the times and changing the repertoire of our symphonic band that was formerly based on orchestral transcriptions, going on to perform original compositions for the band, which gives sonorities sound planes much more successful. These composers-directors who have been at the head of the group, as we can see in the hand programs of the civic-military concerts that we have collected and analyzed, have combined the direction with the composition, premiering different works with this group. This music has always been a reference for all military directors who have wished to one day become director of this prestigious band and is also an image to be followed by civilian bands, because in the history of Spain has always been an extraordinary.

Keywords: Military music; military repertoire; military band

La Unidad de Música de la Guardia Real estuvo dirigida entre 1973 y 1988, por el conquense José López Calvo, al cual le seguiría el alicantino Francisco Grau Vegara, entre 1988 y 2008, seguido éste por el valenciano Antonio Sendra Cebolla entre 2008 y 2010. Durante estos periodos de tiempo se realizaron conciertos cívico-militares que quedaron recogidos en programas de mano, a los cuales hemos podido tener acceso al encontrarse en el archivo de la Unidad de Música de la Guardia Real. Éstos han sido estudiados y analizados en su contenido, mediante la observación y manipulación de datos por medio del programa «Excel», y a continuación se han trasladado al programa «Word», mostrando estos resultados en gráficos y tablas. En la siguiente tabla exponemos la cantidad de programas recogidos desde 1973 hasta 2010. Observamos que muchos de los programas de los primeros años se han perdido, ya que nos remontamos a hace cuarenta y cinco años, durante los cuales hemos podido recopilar seiscientos cincuenta y dos programas.

Tabla 1. Cantidad de programas de conciertos analizados por año

\begin{tabular}{|c|c|c|c|c|c|c|c|c|c|}
\hline 1973 & 1974 & 1975 & 1976 & 1977 & 1978 & 1979 & 1980 & 1981 & 1982 \\
\hline 2 & 0 & 2 & 1 & 4 & 2 & 0 & 2 & 1 & 4 \\
\hline 1983 & 1984 & 1985 & 1986 & 1987 & 1988 & 1989 & 1990 & 1991 & 1992 \\
\hline 5 & 6 & 19 & 23 & 17 & 16 & 24 & 24 & 25 & 26 \\
\hline 1993 & 1994 & 1995 & 1996 & 1997 & 1998 & 1999 & 2000 & 2001 & 2002 \\
\hline 27 & 24 & 22 & 29 & 15 & 26 & 33 & 15 & 31 & 27 \\
\hline 2003 & 2004 & 2005 & 2006 & 2007 & 2008 & 2009 & 2010 & & Total \\
\hline 32 & 24 & 32 & 28 & 26 & 31 & 25 & 4 & & 652 \\
\hline
\end{tabular}




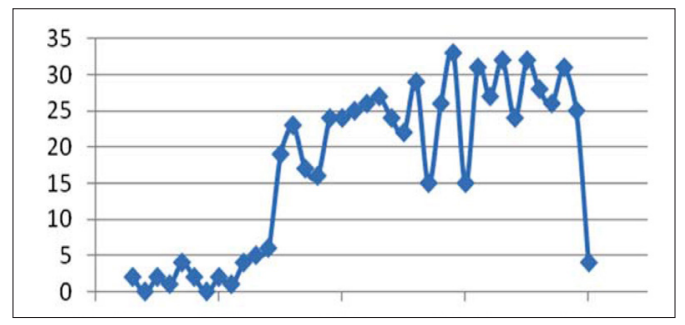

Figura 1. Cantidad de programas de conciertos analizados por año.

El gráfico anterior, muestra la cantidad de programas recogidos (seiscientos cincuenta y dos), según el año de publicación; destacando en 1999 el máximo histórico de los programas recopilados, siendo treinta y tres, mientras que 1974 se puede presentar como el año que menos, ya que no se ha podido recuperar ningún programa correspondiente a este año. Si dividimos los treinta y siete años que abarca este estudio en décadas, observamos que en este último decenio (19992009) se encuentra bastante homogeneidad, no bajando de quince conciertos en 2000 y el máximo en 1999 como dijimos anteriormente, con treinta y tres. En la década anterior (1988-1998), observamos que en el año 1997, el mínimo de conciertos analizados es de quince, mientras que el año anterior 1996 alcanza veintinueve, siendo la mayor producción científica de esta década. La década de menor producción se encuentran entre 1973 y 1983, pero seguramente no es por otra causa más que por que se hayan perdido los programas, no conservando así constancia de la producción de conciertos en estos años.

Después de numerar los programas recopilados, hemos analizado su contenido, dedicando un apartado especial al análisis del género musical, para lo cual, hemos dividido el contenido en tres apartados, un primer apartado donde recogemos la música sinfónica interpretada por la Unidad de Música de la Guardia Real, un segundo apartado, que computa la música popular y de películas ejecutada y un tercer apartado donde se ordena la música militar y pasodobles reseñados.

Tabla 2. Porcentajes de los registros analizados por Categorías de las obras interpretadas en los conciertos

\begin{tabular}{|c|c|c|}
\hline & Género musical & Porcentaje \\
\hline 1 & Música sinfónica & $30,79 \%$ \\
\hline 2 & Música Popular/de Películas & $34,73 \%$ \\
\hline 3 & Mús. Militar/Pasodobles & $34,58 \%$ \\
\hline
\end{tabular}


Con significativa diferencia, en el punto dos, encontramos la música popular y de películas como la categoría más numerosa entre los registros analizados, ya que este apartado comprende zarzuelas, bandas sonoras de películas, arreglos e instrumentaciones de música folklórica para banda sinfónica, siendo el repertorio más interpretado por la Unidad de Música de la Guardia Real en este periodo de tiempo. En cuanto al apartado tres, que sería lo que se supone que una agrupación de música militar, habría de interpretar, con más abundancia es sin embargo el menos numeroso, aunque es el estilo más valorado por nuestro público, el cual suele asistir a los conciertos de bandas militares para escuchar música militar (himnos, marchas militares, selecciones de fragmentos de música castrense y pasodobles). Bien es cierto que siempre se interpreta una marcha militar de bis, como por ejemplo Las Corsarias, conocida popularmente por La Banderita, u otra de arraigado espíritu nacional como La Orgía Dorada. El primer apartado, corresponde a sinfonías, oberturas, fragmentos de ópera, etc., transcritas de orquesta a banda sinfónica. Estas obras sinfónicas, han sido muy comunes en el repertorio de bandas civiles y militares, porque es donde realmente se puede lucir el nivel instrumental y musical de la agrupación, además de popularizarse este género haciéndolo llegar a todos los públicos, fuera de los auditorios. Es más, se trata de una tradición antigua que viene manteniéndose desde las programaciones de 1895, donde se venían interpretando las óperas más importantes del S. XIX. Además en aquella época, eran de habitual interpretación valses vieneses «a nuestra Reina Regente, Austríaca de nacimiento era de obligada cortesía traerle, la música a 3/4, en recuerdo de su país de origen» (Fernández, 1999, p. 355).

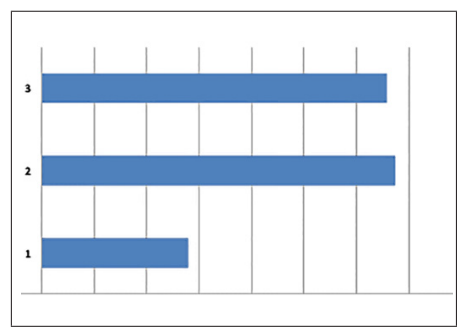

1. Música sinfónica

2. Música Popular/de Películas

3. Mús. Militar/Pasodobles

Figura 2. Categorías de las obras interpretadas en los conciertos.

Es interesante señalar que aunque la música popular y de películas fue el estilo más interpretado, es imprescindible en todos los conciertos, interpretar alguna pieza de música militar, pues el público, al ver la banda uniformada tocando, no duda en pedir marchas militares de bis, siendo la más esperada en todos los eventos, La Banderita, casi como si de un himno representante del Ministerio de Defensa se tratase. 
A continuación, presentamos una división de la programación, según el público al que fue dirigido, atendiendo a publico militar, normalmente dentro de acuartelamientos, en celebraciones patronales o aniversarios de unidades, cambios de mando y despedidas de personal militar. Aunque a estos conciertos suele asistir personal civil, acompañando a familiares o amigos del personal militar, la mayoría de los asistentes son militares, por lo que contamos estos eventos como militares. Los conciertos cívico-militares, son los que se han venido realizando normalmente en plazas públicas, auditorios o salas de concierto, siempre abiertos a todos los públicos y sin cobro de entrada (entrada libre), pues la Unidad de Música de la Guardia Real trabaja sin ánimo de lucro. La organización del concierto suele estar solicitada por ayuntamientos o entidades civiles, que en colaboración con la unidad de protocolo de la Unidad de Música de la Guardia Real consigue establecer conciertos destinados a los habitantes de pueblos y ciudades de todos los lugares donde dispongan esta colaboración.

Tabla 3. Cantidad de programas de conciertos analizados por año según el público al que fue dirigido

CONCIERTOS MILITARES CONCIERTOS CÍVICO-MILITARES

\begin{tabular}{|c|c|c|c|c|c|c|c|c|c|}
\hline 1973 & 1974 & 1975 & 1976 & 1977 & 1978 & 1979 & 1980 & 1981 & 1982 \\
\hline 0 & 0 & 0 & 0 & 0 & 0 & 0 & 0 & 0 & 0 \\
\hline 2 & 0 & 2 & 1 & 4 & 2 & 0 & 2 & 1 & 4 \\
\hline 1983 & 1984 & 1985 & 1986 & 1987 & 1988 & 1989 & 1990 & 1991 & 1992 \\
\hline 0 & 0 & 3 & 9 & 2 & 7 & 9 & 4 & 7 & 6 \\
\hline 5 & 6 & 16 & 14 & 15 & 9 & 15 & 20 & 18 & 20 \\
\hline 1993 & 1994 & 1995 & 1996 & 1997 & 1998 & 1999 & 2000 & 2001 & 2002 \\
\hline 6 & 5 & 6 & 12 & 5 & 9 & 4 & 2 & 1 & 10 \\
\hline 21 & 19 & 16 & 17 & 10 & 17 & 29 & 13 & 30 & 17 \\
\hline 2003 & 2004 & 2005 & 2006 & 2007 & 2008 & 2009 & 2010 & \multirow{2}{*}{\multicolumn{2}{|c|}{$77,76 \% \mathrm{CM}$}} \\
\hline 10 & 5 & 6 & 6 & 2 & 5 & 4 & 1 & & \\
\hline 22 & 19 & 26 & 22 & 24 & 26 & 21 & 3 & \multicolumn{2}{|c|}{$22,24 \% \mathrm{CCM}$} \\
\hline
\end{tabular}

A través de la siguiente tabla podemos resaltar la importancia de la cooperación entre entidad militar y civil, ya que la mayoría de los conciertos realizados por la Música de la Guardia Real han sido destinados a un público civil (77,76\% frente al 22,24\%) y la organización ha estado a cargo de diferentes estamentos civiles que en colaboración con la Sección de Protocolo de la Guardia Real, han organizado diferentes conciertos en plazas y auditorios públicos, siempre sin ánimo de lucro. 


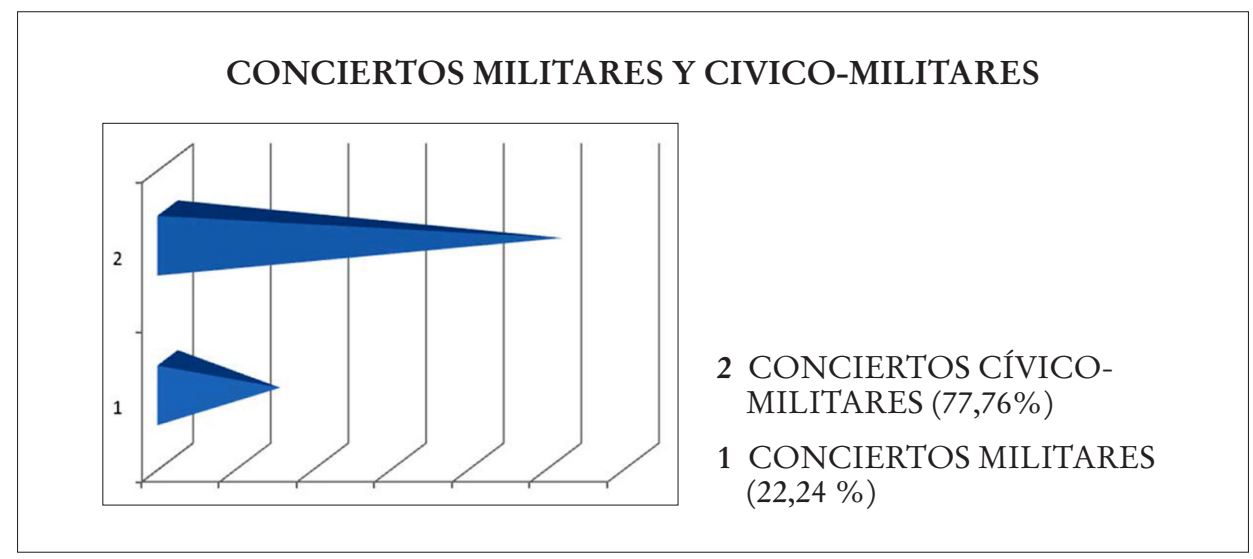

Figura 3. Cantidad de programas de conciertos analizados por año según el público al que fue dirigido.

El siguiente análisis establece una relación entre conciertos nacionales e internacionales; la mayoría de los conciertos realizados por la Unidad de Música de la Guardia Real, son conciertos nacionales (79,60\%). Éstos son los destinados al pueblo español, en toda su geografía. Entendiendo pues, como concierto internacional todos aquellos que aun realizándose dentro de nuestras fronteras han tenido una repercusión internacional, como el caso de los conciertos del Palacio Real de Madrid que Sus Majestades han ofrecido en honor de Jefes de Estado y Monarcas extranjeros (20,40\%).

Las giras de la Unidad de Música de la Guardia Real por el extranjero, también se suman como conciertos internacionales, pero aun así, se puede comprobar que la importancia en representar nuestras músicas fuera de España, ha ido a menos, en el transcurso de los años. Puede ser fruto de las necesidades económicas que reducen las posibilidades de salir a eventos internacionales o el desinterés por parte de los gobiernos en mostrar orgullosamente nuestras bandas de música militares, fuera de nuestras fronteras.

Ahora mostraremos gráficamente esta diferencia, año por año, para observar esta poca representación que comentamos. Las líneas azules, corresponden a conciertos nacionales y las de color naranja a conciertos internacionales. Hay que tener en cuenta que las líneas naranjas correspondientes a los primeros años del muestreo, representan conciertos realizados en el Palacio Real de Madrid, en honor de Jefes de Estado, Monarcas y representantes de otros países invitados a recepciones españolas instituidas en su honor. 
Tabla 4. Cantidad de programas de conciertos analizados por año según el tipo de concierto

CONCIERTOS NACIONALES CONCIERTOS INTERNACIONALES

\begin{tabular}{|c|c|c|c|c|c|c|c|c|c|}
\hline 1973 & 1974 & 1975 & 1976 & 1977 & 1978 & 1979 & 1980 & 1981 & 1982 \\
\hline 0 & 0 & 1 & 0 & 0 & 0 & 0 & 0 & 0 & 0 \\
\hline 2 & 0 & 1 & 1 & 4 & 2 & 0 & 2 & 1 & 4 \\
\hline 1983 & 1984 & 1985 & 1986 & 1987 & 1988 & 1989 & 1990 & 1991 & 1992 \\
\hline 0 & 1 & 7 & 18 & 11 & 11 & 19 & 19 & 21 & 16 \\
\hline 5 & 5 & 12 & 5 & 6 & 5 & 4 & 5 & 4 & 10 \\
\hline 1993 & 1994 & 1995 & 1996 & 1997 & 1998 & 1999 & 2000 & 2001 & 2002 \\
\hline 22 & 17 & 18 & 22 & 12 & 22 & 28 & 13 & 29 & 25 \\
\hline 5 & 7 & 4 & 7 & 3 & 4 & 5 & 2 & 2 & 2 \\
\hline 2003 & 2004 & 2005 & 2006 & 2007 & 2008 & 2009 & 2010 & \multirow{3}{*}{\multicolumn{2}{|c|}{$\begin{array}{l}79,60 \% \mathrm{CM} \\
20,40 \% \mathrm{CI}\end{array}$}} \\
\hline 31 & 23 & 31 & 26 & 22 & 29 & 22 & 4 & & \\
\hline 1 & 1 & 1 & 2 & 4 & 2 & 3 & 0 & & \\
\hline
\end{tabular}

\section{CONCIERTOS NACIONALES E INTERNACIONALES}

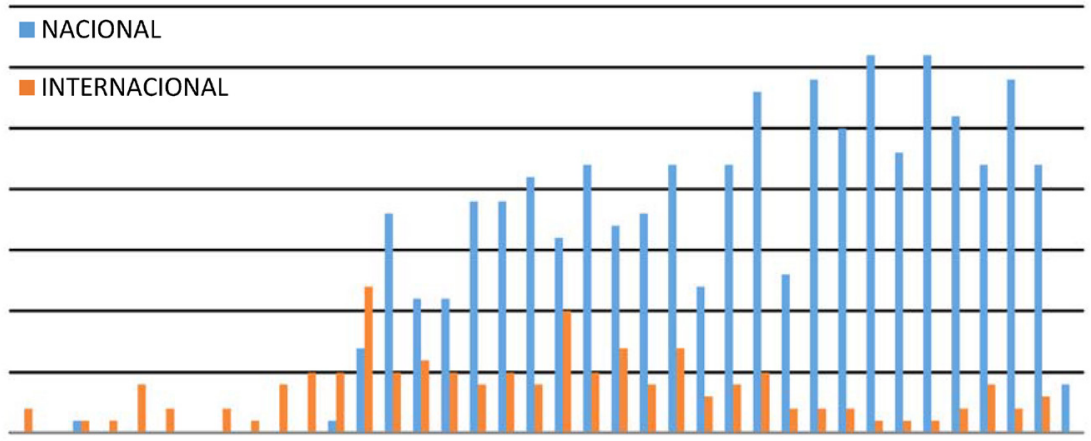

Figura 4. Cantidad de programas de conciertos analizados por año según el tipo de concierto

España se compone de cincuenta provincias (Álava, Albacete, Alicante, Almería, Ávila, Badajoz, Barcelona, Burgos, Cáceres, Cádiz, Castellón, Ciudad Real, Córdoba, La Coruña, Cuenca, Gerona, Granada, Guadalajara, Guipúzcoa, Huelva, Huesca, Islas Baleares, Jaén, Las Palmas, León, Lérida, Logroño, Lugo, 
Madrid, Málaga, Murcia, Orense, Oviedo, Palencia, Pamplona, Pontevedra, Salamanca, Santander, Segovia, Sevilla, Soria, Tarragona, Tenerife, Teruel, Toledo, Valencia, Valladolid, Vizcaya, Zamora, Zaragoza) y dos ciudades autónomas (Ceuta y Melilla).

Dentro de nuestras fronteras, la Unidad de Música de la Guardia Real, ha actuado en casi todas las comunidades autónomas y la comunidad foral de Navarra, además de las ciudades autónomas de Ceuta y Melilla. Resultando de ello que han viajado a Andalucía, Aragón, Asturias, Castilla la Mancha, Castilla y León, Cataluña, Ceuta, Comunidad Valenciana, Extremadura, Galicia, Canarias, Comunidad de Madrid, Melilla, Región de Murcia y Navarra. Y siendo cuatro comunidades autónomas, las que no han recibido a la Música de la Unidad de Música de la Guardia Real en este periodo de tiempo, Cantabria, Islas Baleares, La Rioja y País Vasco.

De entre todas las provincias extranjeras, la Unidad de Música de la Guardia Real ha visitado Bonn, Bruselas, Oslo, Turín (Alemania, Bélgica, Noruega e Italia), realizando el número de conciertos, que a continuación figuran, resultando los porcentajes siguientes:

Tabla 5. Totales y porcentajes de los registros analizados por Lugar (Ciudades) de realización del concierto.

\section{CIUDADES DONDE SE HAN REALIZADO LOS CONCIERTOS}

\begin{tabular}{|l|c|c|}
\hline \multicolumn{1}{|c|}{ LUGAR } & TOTAL & $\%$ \\
\hline Albacete & 1 & 0,15 \\
\hline Alicante & 6 & 0,92 \\
\hline Almería & 2 & 0,31 \\
\hline Ávila & 1 & 0,15 \\
\hline Barcelona & 2 & 0,31 \\
\hline Bonn (Alemania) & 1 & 0,15 \\
\hline Bruselas (Bélgica) & 1 & 0,15 \\
\hline Burgos & 3 & 0,46 \\
\hline Cádiz & 2 & 0,31 \\
\hline Canarias & 3 & 0,46 \\
\hline Cartagena (Murcia) & 1 & 0,15 \\
\hline Castellón & 2 & 0,31 \\
\hline Ceuta & 2 & 0,31 \\
\hline Ciudad Real & 1 & 0,15 \\
\hline Córdoba & 3 & 0,46 \\
\hline
\end{tabular}

\begin{tabular}{|l|c|c|}
\hline \multicolumn{1}{|c|}{ LUGAR } & TOTAL & $\%$ \\
\hline Coruña & 2 & 0,31 \\
\hline Cuenca & 1 & 0,15 \\
\hline Figueres (Gerona) & 1 & 0,15 \\
\hline Granada & 4 & 0,61 \\
\hline Huesca & 1 & 0,15 \\
\hline Jaén & 1 & 0,15 \\
\hline Jerez (Cádiz) & 1 & 0,15 \\
\hline León & 2 & 0,31 \\
\hline Lérida & 1 & 0,15 \\
\hline Lugo & 1 & 0,15 \\
\hline Madrid & 552 & 87,12 \\
\hline Málaga & 1 & 0,15 \\
\hline Melilla & 1 & 0,15 \\
\hline Murcia & 1 & 0,15 \\
\hline Orense & 1 & 0,15 \\
\hline
\end{tabular}




\begin{tabular}{|l|c|c|}
\hline \multicolumn{1}{|c|}{ LUGAR } & TOTAL & $\%$ \\
\hline Oslo (Noruega) & 1 & 0,15 \\
\hline Oviedo & 2 & 0,31 \\
\hline Pamplona & 1 & 0,15 \\
\hline Segovia & 11 & 1,69 \\
\hline Sevilla & 3 & 0,46 \\
\hline Soria & 1 & 0,15 \\
\hline Tenerife & 1 & 0,15 \\
\hline
\end{tabular}

\begin{tabular}{|l|c|c|}
\hline \multicolumn{1}{|c|}{ LUGAR } & TOTAL & $\%$ \\
\hline Teruel & 1 & 0,15 \\
\hline Toledo & 6 & 0,92 \\
\hline Torrevieja (Alicante) & 1 & 0,15 \\
\hline Turín & 2 & 0,31 \\
\hline Valencia & 2 & 0,31 \\
\hline Zamora & 1 & 0,15 \\
\hline Zaragoza & 1 & 0,15 \\
\hline
\end{tabular}

La Música de la Guardia Real no ha podido acercar sus sonidos a todas las provincias españolas, quedando las siguientes provincias españolas sin conciertos durante este periodo de tiempo; Badajoz, Cáceres, Guadalajara, Guipúzcoa, Huelva, Islas Baleares, Logroño, Palencia, Pontevedra, Salamanca, Santander, Tarragona, Valladolid y Vizcaya, además hay tres conciertos que figuran como que están realizados en Canarias, pero no sabemos si en la provincias de Las Palmas, en la de Santa Cruz de Tenerife, ya que no aparece especificado en el programa de mano. Por lo que creemos que no ha podido actuar en todo el archipiélago canario; Fuerteventura, Lanzarote, Tenerife, La Palma, La Gomera, o El Hierro.

A continuación observamos un gráfico, donde podemos ver los detalles de los porcentajes de los conciertos realizados en Madrid (87,12\%) y el resto de ciudades españolas y extranjeras, que suman entre todas el $12,88 \%$.

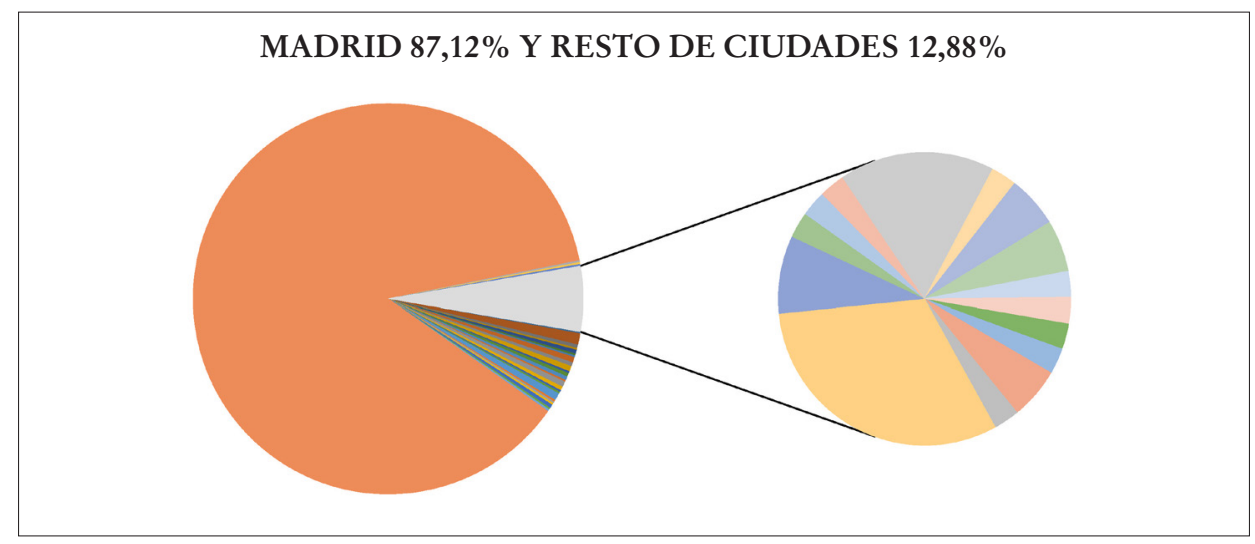

Figura 5. Porcentajes de los registros analizados por Lugar de realización del concierto

La mayoría de los conciertos realizados por la Unidad de Música de la Guardia Real, se han interpretado en Madrid, un $87,12 \%$, quinientos sesenta y nueve 
conciertos de seiscientos cincuenta y dos programas que se han analizado. El resto, ochenta y tres programas se han podido recoger de conciertos realizados en diferentes ciudades de España y del extranjero, sumando entre todas ellas el $12,88 \%$ restante. Después de Madrid, la ciudad que más ha acogido a la agrupación musical de la Guardia Real, ha sido Segovia, con once conciertos, seguida de Alicante y Toledo, con seis cada una. Granada será la siguiente afortunada que pudo disfrutar de cuatro conciertos, tres en 1990 y uno en 2007. Las ciudades que menos han podido disfrutar de los conciertos de la Guardia Real, aun teniendo la suerte de tenerlas en un 0,15\% fueros Albacete (2003), Ávila (2007), Bonn (1986), Bruselas (1992), Cartagena (1999), Ciudad Real (2005), Cuenca (2009), Figueres (2003), Huesca (1992), Jaén (2008), Jerez (1993), Lérida (1991), Lugo (1995), Málaga (2006), Melilla (2002), Murcia (1995), Orense (1995), Oslo (2006), Pamplona (1996), Soria (2004), Tenerife (2003), Teruel (2003), Torrevieja (2006), Zamora (2009), y Zaragoza (2008). Hasta dos veces pudieron recibirla, alcanzando un $0,31 \%$ en Almería (2004), Barcelona (2001 y 2003), Cádiz (1993), Castellón (2002), Ceuta (1999), Coruña (2005 y 2006), León (1996 y 2007), Oviedo (1984 y 2003), Turín (1992) y Valencia (2003 y 2006). Con 0,46\% pudieron oír esta agrupación hasta tres veces en Burgos (1993, 2002 y 2006) y tres en Canarias en 1986.

Para poder detenerse a examinar estos detalles, presentamos diferentes tablas estructuradas por décadas, la primera tabla enmarca desde 1973 a 1982.

Tabla 6. Primera década (1973 - 1982)

\begin{tabular}{|l|l|l|l|l|l|l|l|l|l|l|}
\hline \multicolumn{1}{|c|}{ AÑ } & 1973 & 1974 & 1975 & 1976 & 1977 & 1978 & 1979 & 1980 & 1981 & 1982 \\
\hline Albacete & & & & & & & & & & \\
\hline Alicante & & & & & & & & & & \\
\hline Almería & & & & & & & & & & \\
\hline Ávila & & & & & & & & & & \\
\hline Barcelona & & & & & & & & & & \\
\hline Bonn & & & & & & & & & & \\
\hline Bruselas & & & & & & & & & & \\
\hline Burgos & & & & & & & & & & \\
\hline Cádiz & & & & & & & & & & \\
\hline Canarias & & & & & & & & & & \\
\hline Cartagena & & & & & & & & & & \\
\hline Castellón & & & & & & & & & & \\
\hline Ceuta & & & & & & & & & & \\
\hline Ciudad Real & & & & & & & & & & \\
\hline
\end{tabular}


EVA FOLCH MARTÍNEZ

PROGRAMACIÓN DE LA UNIDAD DE MÚSICA DE LA GUARDIA REAL ENTRE 1973 Y 2010

\begin{tabular}{|l|l|l|l|l|l|l|l|l|l|l|}
\hline \multicolumn{1}{|c|}{ AÑO } & 1973 & 1974 & 1975 & 1976 & 1977 & 1978 & 1979 & 1980 & 1981 & 1982 \\
\hline Córdoba & & & & & & & & & & \\
\hline Coruña & & & & & & & & & & \\
\hline Cuenca & & & & & & & & & & \\
\hline Figueres & & & & & & & & & & \\
\hline Granada & & & & & & & & & & \\
\hline Huesca & & & & & & & & & & \\
\hline Jaén & & & & & & & & & & \\
\hline Jerez & & & & & & & & & & \\
\hline León & & & & & & & & & & \\
\hline Lérida & & & & & & & & & & \\
\hline Lugo & & & & & & & & & & \\
\hline Madrid & 2 & & 2 & 1 & 2 & 2 & & 2 & 1 & 4 \\
\hline Málaga & & & & & & & & & & \\
\hline Melilla & & & & & & & & & & \\
\hline Murcia & & & & & & & & & & \\
\hline Orense & & & & & & & & & & \\
\hline Oslo & & & & & & & & & & \\
\hline Oviedo & & & & & & & & & & \\
\hline Pamplona & & & & & & & & & & \\
\hline Segovia & & & & & & & & & & \\
\hline Sevilla & & & & & & & & & & \\
\hline Soria & & & & & & & & & & \\
\hline Tenerife & & & & & & & & & & \\
\hline Teruel & & & & & & & & & & \\
\hline Toledo & & & & & & & & & & \\
\hline Torrevieja & & & & & & & & & & \\
\hline Turín & & & & & & & & & & \\
\hline Valencia & & & & & & & & & & \\
\hline Zamora & & & & & & & & & & \\
\hline Zaragoza & & & & & & & & & & \\
\hline
\end{tabular}

Ediciones Universidad de Salamanca /

Popular Music Research Today, pp. 69-87 
EVA FOLCH MARTÍNEZ

PROGRAMACIÓN DE LA UNIDAD DE MÚSICA DE LA GUARDIA REAL ENTRE 1973 Y 2010

La siguiente tabla encuadra los años desde 1983 a 1992:

Tabla 7. Segunda década (1983 - 1992)

\begin{tabular}{|l|l|l|l|l|l|l|l|l|l|l|}
\hline \multicolumn{1}{|c|}{ AÑO } & 1983 & 1984 & 1985 & 1986 & 1987 & 1988 & 1989 & 1990 & 1991 & 1992 \\
\hline Albacete & & & & & & & & & & \\
\hline Alicante & & & & & & & 1 & 1 & & \\
\hline Almería & & & & & & & & & & \\
\hline Ávila & & & & & & & & & & \\
\hline Barcelona & & & & & & & & & & \\
\hline Bonn & & & & 1 & & & & & & \\
\hline Bruselas & & & & & & & & & & 1 \\
\hline Burgos & & & & & & & & & & \\
\hline Cádiz & & & & & & & & & & \\
\hline Canarias & & & & 3 & & & & & & \\
\hline Cartagena & & & & & & & & & & \\
\hline Castellón & & & & & & & & & & \\
\hline Ceuta & & & & & & & & & & \\
\hline Ciudad Real & & & & & & & & & & \\
\hline Córdoba & & & & & & & & & & \\
\hline Coruña & & & & & & & & & & \\
\hline Cuenca & & & & & & & & & & \\
\hline Figueres & & & & & & & & & & \\
\hline Granada & & & & & & & & & 3 & \\
\hline Huesca & & & & & & & & & & 1 \\
\hline Jaén & & & & & & & & & & \\
\hline Jerez & & & & & & & & & & \\
\hline León & & & & & & & & & & \\
\hline Lérida & & & & & & & & & 1 & \\
\hline Lugo & & & & & & & & & & \\
\hline Madrid & 5 & 5 & 19 & 18 & 17 & 15 & 22 & 23 & 20 & 21 \\
\hline Málaga & & & & & & & & & & \\
\hline Melilla & & & & & & & & & & \\
\hline Murcia & & & & & & & & & & \\
\hline Orense & & & & & & & & & & \\
\hline Oslo & & & & & & & & & & \\
\hline Oviedo & & 1 & & & & & & & & \\
\hline & & & & & & & \\
\hline
\end{tabular}

Ediciones Universidad de Salamanca /

Popular Music Research Today, pp. 69-87 
EVA FOLCH MARTÍNEZ

PROGRAMACIÓN DE LA UNIDAD DE MÚSICA DE LA GUARDIA REAL ENTRE 1973 Y 2010

\begin{tabular}{|l|l|l|l|l|l|l|l|l|l|l|}
\hline \multicolumn{1}{|c|}{ AÑO } & 1983 & 1984 & 1985 & 1986 & 1987 & 1988 & 1989 & 1990 & 1991 & 1992 \\
\hline Pamplona & & & & & & & & & & \\
\hline Segovia & & & & & & 1 & & & & \\
\hline Sevilla & & & & & & & & & 1 & 1 \\
\hline Soria & & & & & & & & & & \\
\hline Tenerife & & & & & & & & & & \\
\hline Teruel & & & & & & & & & & \\
\hline Toledo & & & & & & & & & & \\
\hline Torrevieja & & & & & & & & & & \\
\hline Turín & & & & & & & & & & 2 \\
\hline Valencia & & & & & & & & & & \\
\hline Zamora & & & & & & & & & & \\
\hline Zaragoza & & & & & & & & & & \\
\hline
\end{tabular}

Esta tabla recoge los datos desde 1993 a 2002:

Tabla 8. Tercera década (1993 - 2002)

\begin{tabular}{|l|c|c|c|c|c|c|c|c|c|c|}
\hline \multicolumn{1}{|c|}{ AÑ } & 1993 & 1994 & 1995 & 1996 & 1997 & 1998 & 1999 & 2000 & 2001 & 2002 \\
\hline Albacete & & & & & & & & & & \\
\hline Alicante & 2 & 1 & & & & & & & & \\
\hline Almería & & & & & & & & & & \\
\hline Ávila & & & & & & & & & & \\
\hline Barcelona & & & & & & & & & 1 & \\
\hline Bonn & & & & & & & & & & \\
\hline Bruselas & & & & & & & & & & \\
\hline Burgos & 1 & & & & & & & & & 1 \\
\hline Cádiz & 2 & & & & & & & & & \\
\hline Canarias & & & & & & & & & & \\
\hline Cartagena & & & & & & & 1 & & & \\
\hline Castellón & & & & & & & & & & 2 \\
\hline Ceuta & & & & & & & 2 & & & \\
\hline Ciudad Real & & & & & & & & & & \\
\hline Córdoba & & & 2 & & & & & & & \\
\hline Coruña & & & & & & & & & & \\
\hline Cuenca & & & & & & & & & & \\
\hline Figueres & & & & & & & & & & \\
\hline
\end{tabular}

Ediciones Universidad de Salamanca /

Popular Music Research Today, pp. 69-87 
EVA FOLCH MARTÍNEZ

PROGRAMACIÓN DE LA UNIDAD DE MÚSICA DE LA GUARDIA REAL ENTRE 1973 Y 2010

\begin{tabular}{|l|c|c|c|c|c|c|c|c|c|c|}
\hline \multicolumn{1}{|c|}{ AÑO } & 1993 & 1994 & 1995 & 1996 & 1997 & 1998 & 1999 & 2000 & 2001 & 2002 \\
\hline Granada & & & & & & & & & & \\
\hline Huesca & & & & & & & & & & \\
\hline Jaén & & & & & & & & & & \\
\hline Jerez & 1 & & & & & & & & & \\
\hline León & & & & 1 & & & & & & \\
\hline Lérida & & & & & & & & & & \\
\hline Lugo & & & 1 & & & & & & & \\
\hline Madrid & 20 & 23 & 17 & 27 & 14 & 25 & 29 & 14 & 30 & 21 \\
\hline Málaga & & & & & & & & & & \\
\hline Melilla & & & & & & & & & & 1 \\
\hline Murcia & & & 1 & & & & & & & \\
\hline Orense & & & 1 & & & & & & & \\
\hline Oslo & & & & & & & & & & \\
\hline Oviedo & & & & & & & & & & \\
\hline Pamplona & & & & 1 & & & & & & \\
\hline Segovia & & & & & & 1 & 1 & 1 & & 1 \\
\hline Sevilla & & & & & & & & & & \\
\hline Soria & & & & & & & & & & \\
\hline Tenerife & & & & & & & & & & \\
\hline Teruel & & & & & & & & & & \\
\hline Toledo & 1 & & & & 1 & & & & & 1 \\
\hline Torrevieja & & & & & & & & & & \\
\hline Turín & & & & & & & & & & \\
\hline Valencia & & & & & & & & & & \\
\hline Zamora & & & & & & & & & & \\
\hline Zaragoza & & & & & & & & & & \\
\hline
\end{tabular}

La última tabla recoge los datos desde 2003 a 2010, además de los totales y porcentaje de todas las tablas anteriores, sumando los programas desde 1973 hasta 2010:

Tabla 9. Últimos años (2003-2010)

\begin{tabular}{|l|c|c|c|c|c|c|c|c|c|c|}
\hline \multicolumn{1}{|c|}{ AÑ } & 2003 & 2004 & 2005 & 2006 & 2007 & 2008 & 2009 & 2010 & Total & Porcentaje \\
\hline Albacete & 1 & & & & & & & & 1 & 0,01 \\
\hline Alicante & & & 1 & & & & & & 6 & 0,06 \\
\hline Almería & & 2 & & & & & & & 2 & 0,02 \\
\hline
\end{tabular}

Ediciones Universidad de Salamanca / 
EVA FOLCH MARTÍNEZ

PROGRAMACIÓN DE LA UNIDAD DE MÚSICA DE LA GUARDIA REAL ENTRE 1973 Y 2010

\begin{tabular}{|l|c|c|c|c|c|c|c|c|c|c|}
\hline \multicolumn{1}{|c|}{ AÑO } & 2003 & 2004 & 2005 & 2006 & 2007 & 2008 & 2009 & 2010 & Total & Porcentaje \\
\hline Ávila & & & & & 1 & & & & 1 & 0,01 \\
\hline Barcelona & 1 & & & & & & & & 2 & 0,02 \\
\hline Bonn & & & & & & & & & 1 & 0,01 \\
\hline Bruselas & & & & & & & & & 1 & 0,01 \\
\hline Burgos & & & & 1 & & & & & 3 & 0,03 \\
\hline Cádiz & & & & & & & & & 2 & 0,02 \\
\hline Canarias & & & & & & & & & 3 & 0,03 \\
\hline Cartagena & & & & & & & & & 1 & 0,01 \\
\hline Castellón & & & & & & & & & 2 & 0,02 \\
\hline Ceuta & & & & & & & & & 2 & 0,02 \\
\hline Ciudad Real & & & 1 & & & & & & 1 & 0,01 \\
\hline Córdoba & & & & & & 1 & & & 3 & 0,03 \\
\hline Coruña & & & 1 & 1 & & & & & 2 & 0,02 \\
\hline Cuenca & & & & & & & 1 & & 1 & 0,01 \\
\hline Figueres & 1 & & & & & & & & 1 & 0,01 \\
\hline Granada & & & & & 1 & & & & 4 & 0,04 \\
\hline Huesca & & & & & & & & & 1 & 0,01 \\
\hline Jaén & & & & & & 1 & & & 1 & 0,01 \\
\hline Jerez & & & & & & & & & 1 & 0,01 \\
\hline León & & & & & 1 & & & & 2 & 0,02 \\
\hline Lérida & & & & & & & & & 1 & 0,01 \\
\hline Lugo & & & & & & & & & 1 & 0,01 \\
\hline Madrid & 25 & 21 & 28 & 20 & 21 & 25 & 23 & 4 & 552 & 5,52 \\
\hline Málaga & & & & 1 & & & & & 1 & 0,01 \\
\hline Melilla & & & & & & & & & 1 & 0,01 \\
\hline Murcia & & & & & & & & & 1 & 0,01 \\
\hline Orense & & & & & & & & & 1 & 0,01 \\
\hline Oslo & & & & 1 & & & & & 1 & 0,01 \\
\hline Oviedo & 1 & & & & & & & & 2 & 0,02 \\
\hline Pamplona & & & & & & & & & 1 & 0,01 \\
\hline Segovia & 1 & & 1 & 1 & 1 & 2 & & & 11 & 0,11 \\
\hline Sevilla & & & & 1 & & & & & 3 & 0,03 \\
\hline Soria & & 1 & & & & & & & 1 & 0,01 \\
\hline Tenerife & 1 & & & & & & & & 1 & 0,01 \\
\hline Teruel & 1 & & & & & & & & 1 & 0,01 \\
\hline
\end{tabular}

Ediciones Universidad de Salamanca /

Popular Music Research Today, pp. 69-87 
EVA FOLCH MARTÍNEZ

PROGRAMACIÓN DE LA UNIDAD DE MÚSICA DE LA GUARDIA REAL ENTRE 1973 Y 2010

\begin{tabular}{|l|c|c|c|c|c|c|c|c|c|c|}
\hline \multicolumn{1}{|c|}{ AÑ } & 2003 & 2004 & 2005 & 2006 & 2007 & 2008 & 2009 & 2010 & Total & Porcentaje \\
\hline Toledo & 1 & & & & 1 & 1 & & & 6 & 0,06 \\
\hline Torrevieja & & & & 1 & & & & & 1 & 0,01 \\
\hline Turín & & & & & & & & & 2 & 0,02 \\
\hline Valencia & 1 & & & 1 & & & & & 2 & 0,02 \\
\hline Zamora & & & & & & & 1 & & 1 & 0,01 \\
\hline Zaragoza & & & & & & 1 & & & 1 & 0,01 \\
\hline
\end{tabular}

Tras haber analizado punto por punto, todo el contenido de los programas, numero de programas recogidos, año de publicación, lugar de publicación, género musical, tipo de concierto (militar o cívico-militar), público al que fue dirigido (civil o militar), a continuación nos referiremos a un índice que hemos elaborado, para comparar los tres periodos de programación de la Unidad de Música de la Guardia Real. El primer periodo, fue el del comandante músico José López Calvo, comprendido entre octubre de 1973 y mayo de 1988, el segundo periodo, fue el del teniente coronel músico Francisco Grau Vegara, comprendió desde mayo de 1988, hasta enero de 2008; por último, el tercer periodo, estuvo contenido entre enero de 2008 y junio 2010, bajo la dirección del coronel músico Antonio Sendra Cebolla.

De entre las obras analizadas del periodo del comandante músico José López Calvo, que estuvo de director de la Unidad de Música de la Guardia Real desde que fuera capitán y durante quince años, hemos advertido que la pieza que más se programó fue La Gran Vía del maestro Chueca, interpretándola once veces; a esta le sigue Fiesta en España de Moya, interpretada diez veces y nueve veces El Sitio de Zaragoza de Oudrid. En cambio, al llegar a la dirección el comandante músico Francisco Grau Vegara, el cual se convertiría en general de brigada del Cuerpo de Músicas Militares tras los veinte años de su dirección en esta agrupación, en la cual, vemos respecto a su programación, que la obra más escuchada; setenta y seis veces, sería El Sitio de Zaragoza, con una versión instrumentada por él mismo; después le sigue Mosaico Lírico, con adaptación propia, interpretada cuarenta y seis veces y otra composición suya, programada cuarenta y tres veces; Ecos de una Contienda. Se nota que la intención del siguiente director, el coronel músico Antonio Sendra Cebolla, fue variar más la programación, por lo que en los dos años de conciertos que realizó el que llegase al empleo de coronel músico, sería la obra que más fue interpretada, también El sitio de Zaragoza, pero solo ocho veces, Pompa y Circunstancia de Elgar, seis veces y Doña Francisquita de Vives, cinco veces.

Del análisis de los programas recogidos del archivo de la Guardia Real correspondientes al periodo comprendido entre Octubre de 1973 y junio de 2010, hemos 
extraído un índice compuesto por mil doscientas cincuenta y una obras diferentes interpretadas por esta magnífica Banda Sinfónica de la Guardia Real en este periodo de treinta y siete años. Para establecer mayor entendimiento del análisis de estos programas pertenecientes a estos tres directores que han estado al cargo de la agrupación, hablaremos de tres diferentes etapas de dirección; la primera, bajo la dirección del Maestro Calvo, la segunda, bajo la jefatura de Francisco Grau y la tercera bajo la batuta de Antonio Sendra Cebolla.

En la programación de la Unidad de Música de la Guardia Real entre 1973 y 2010, podemos observar que José López Calvo interpretó trescientas cincuenta y dos obras diferentes en quince años, Francisco Grau Vegara reprodujo ochocientas setenta y tres obras diferentes en veinte años y Antonio Sendra Cebolla reveló trescientas sesenta y una obras diferentes en dos años. Del mismo modo podemos observar que el compositor más interpretado ha sido Francisco Grau con doscientas setenta y ocho piezas originales o arreglos, que analizando por separado los periodos podemos ver que en el periodo de José López Calvo como director se interpretaron dos obras de Francisco Grau, mientras que en el periodo de Francisco Grau como director se interpretaron doscientas setenta y siete diferentes obras suyas dirigidas con la propia batuta del compositor y en el periodo de Antonio Sendra fueron interpretadas diez veces piezas de Francisco Grau. Así mismo observamos que se han interpretado cuarenta y una obras diferentes del Maestro Calvo entre los tres periodos, de las cuales veinticinco fueron interpretadas por él mismo en el primer periodo, veintisiete obras diferentes suyas en el segundo periodo y doce en el tercer periodo. En cuanto a obras de Antonio Sendra podemos observar que se han interpretado treinta y cinco obras diferentes; dos en la época del Maestro Calvo, ninguna con Francisco Grau y cuarenta y una por él mismo. Con estos datos llegamos a la conclusión de que la composición ha sido para los directores de la Guardia Real un tema fuerte y que se han servido de la Banda para darse a conocer como compositores.

Como conclusión a este trabajo, diremos que la Unidad de Música de la Guardia Real ha representado a la música militar española, con un alto nivel artístico, dentro y fuera de nuestras fronteras. En los años 80, intervino en el afamado programa televisivo «Gente Joven», estuvo invitada en el Certamen Internacional de Valencia en 1998, como clausura de la sección especial, interpretando un arreglo de M. González, sobre la banda sonora de Independen Day. Intervinieron en festivales militares en diferentes ocasiones, como en Mons, viajando a Bruselas en 1984, Londres en 1989, Lisboa en 1989, Praga en 2000, París en 2001, Niza en 2002, Colonia en 2004, Turín en 2005, Oslo en 2006 y Bucarest en 2007. Dentro de España, donde más ha estado (seis veces) ha sido en la ciudad de Alicante, entre 1989 y 2005 (coincidiendo con la dirección de Francisco Grau, ya que su ciudad natal Bigastro, pertenece a Alicante), además llevó la Música popular de 
habaneras, interpretadas por corales alicantinas hasta Madrid, grabando un disco con la colaboración del Coro del Maestro Vallejo (Torrevieja, Alicante), bajo el título «Música para una conmemoración» en 2007 con motivo del 30 aniversario del Ministerio de Defensa.

Con el comandante José López Calvo, al frente de esta prestigiosa agrupación, han conseguido honores y felicitaciones dentro y fuera del país. La labor pedagógica del maestro Calvo, ha dado su fruto, convirtiendo en sucesor suyo como directores de esta magnífica agrupación, a Antonio Sendra Cebolla, quien fue alumno suyo y componente de la agrupación musical como Suboficiales Músicos, con las especialidades de Bombardino.

En resumen, sobre el estilo más interpretado durante el periodo que abarcamos, en cuanto a lo que de programación se refiere, ha sido la música militar, representada con un total de noventa y seis veces, la pieza más repentizada durante estos treinta y siete años, El Sitio de Zaragoza.

\section{REFERENCIAS BIBLIOGRÁFICAS}

Fernández, R. (1999). Historia de la Música Militar de España. Madrid: Ministerio de Defensa. Secretaría General Técnica.

Folch, E. (2009). La Unidad de Música de la Guardia Real, antigua de Alabarderos: Treinta y siete años de conciertos. (Máster en educación musical «una perspectiva multidisciplinar») Universidad de Granada. Granada. 